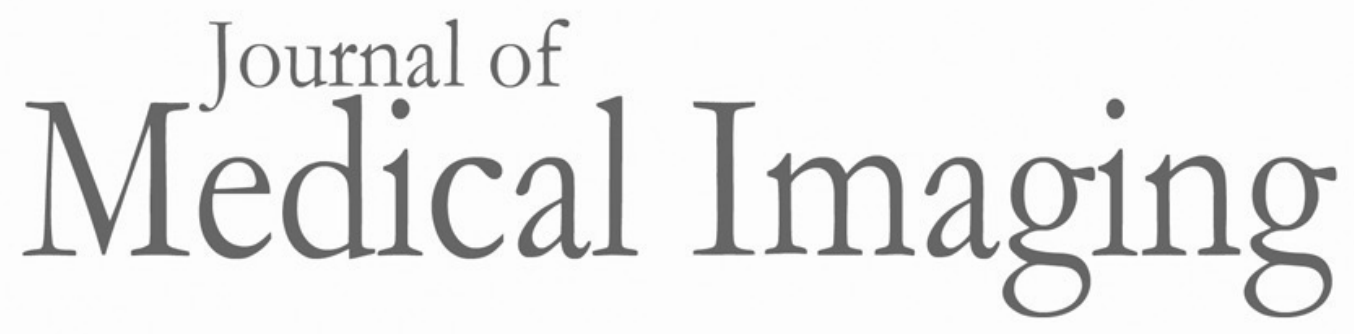

Medicallmaging.SPIEDigitalLibrary.org

\title{
Integrating structured abstracts in the Journal of Medical Imaging
}

Maryellen Giger

SPE Maryellen Giger, "Integrating structured abstracts in the Journal of Medical Imaging," J. Med. Imag. 7(1), 010101 (2020), doi: 10.1117/1.JMI.7.1.010101 


\title{
Integrating structured abstracts in the Journal of Medical Imaging
}

\author{
Maryellen Giger \\ Editor-in-Chief
}

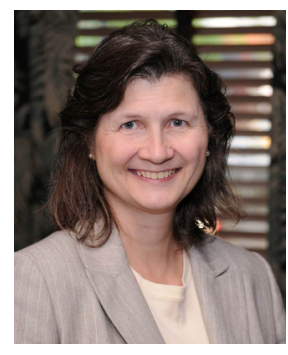

While in the past, the format of abstracts in the Journal of Medical Imaging (JMI) has been left to the authors, the editorial board of JMI has decided that JMI will now require structured abstracts for all submitted manuscripts.

Structured abstracts concisely present the background and significance, aims, scientific approach, results, and conclusions of the research so that readers can efficiently find the papers that are most aligned to their interest and needs. Having these key information components in a structured abstract also aids search engines in identifying appropriate papers.

In addition, it has been shown that papers with structured abstracts are more often accessed online, ${ }^{1,2}$ which is essential in today's overloaded world of publications. It has also been found, in objective assessments, that the quality was higher in structured abstracts than in non-structured abstracts. ${ }^{3}$

The structured JMI abstract should be a summary of the paper within a 250-word maximum. It should be self-contained and substantive, presenting concisely the following four categories:

Purpose: This section presents the significance and aims, stating the broad impact and the rationale or motivation of the work, including potentially some background.

Approach: This section briefly describes the materials and methods, including the statistical analyses, used in the research.

Results: This section provides a core summary of the findings from the work, including study numbers, quantitative analyses, or discoveries.

Conclusions: This section summarizes and interprets the approach and findings from the work, stating also the importance or impact of the findings.

Note that a structured abstract serves as a summary of a paper, and is not just a short introduction.

For further understanding, here is an example of a structured abstract from a recently accepted JMI manuscript: ${ }^{4}$

Purpose: Data-intensive modeling could provide insight on the broad variability in outcomes in spine surgery. Previous studies were limited to analysis of demographic and clinical characteristics. We report an analytic framework called "SpineCloud" that incorporates quantitative features extracted from perioperative images to predict spine surgery outcome.

Approach: A retrospective study was conducted in which patient demographics, imaging, and outcome data were collected. Image features were automatically computed from perioperative CT. Postoperative 3- and 12-month functional and pain outcomes were analyzed in terms of improvement relative to the preoperative state. A boosted decision tree classifier was trained to predict outcome using demographic and image features as predictor variables. Predictions were computed based on SpineCloud and conventional demographic models, and features associated with poor outcome were identified from weighting terms evident in the boosted tree.

Results: Neither approach was predictive of 3- or 12-month outcomes based on preoperative data alone in the current, preliminary study. However, SpineCloud predictions incorporating image features obtained during and immediately following surgery (i.e., intraoperative and immediate postoperative images) exhibited significant improvement in area under the receiver operating characteristic (AUC): $\mathrm{AUC}=0.72\left(\mathrm{CI}_{95}=0.59\right.$ to 0.83$)$ at 3 months and $\mathrm{AUC}=0.69\left(\mathrm{CI}_{95}=0.55\right.$ to 0.82$)$ at 12 months. 
Conclusions: Predictive modeling of lumbar spine surgery outcomes was improved by incorporation of image-based features compared to analysis based on conventional demographic data. The SpineCloud framework could improve understanding of factors underlying outcome variability and warrants further investigation and validation in a larger patient cohort.

Exceptions to the structured abstract format will be left to the authors of JMI opinion/editorial and review papers.

Other SPIE journals have already implemented structured abstracts in their manuscript formats, including the Journal of Biomedical Optics (JBO), Neurophotonics, the Journal of Micro/ Nanolithography, MEMS, and MOEMS, and Optical Engineering. ${ }^{5-7}$ Given that our authors and readers are familiar with scientific abstracts and writing, we expect this switch to required structured abstracts to be welcomed and straightforward.

\section{References}

1. A. M. Ripple et al., "A retrospective cohort study of structured abstracts in MEDLINE, 19922006," J. Med. Libr. Assoc. 99(2), 160-163 (2011).

2. A. M. Harbourt, L. S. Knecht, and B. L. Humphreys, "Structured abstracts in MEDLINE, 1989-1991," Bull. Med. Libr. Assoc. 83(2), 190-195 (1995).

3. A. Taddio et al., "Quality of nonstructured and structured abstracts of original research articles in the British Medical Journal, the Canadian Medical Association Journal and the Journal of the American Medical Association," CMAJ 150(10), 1611-1615 (1994).

4. T. De Silva et al., "SpineCloud: image analytics for predictive modeling of spine surgery outcomes," J. Med. Imaging, 7(2), accepted (2020).

5. B. W. Pogue, "Structured abstracts: the time has come for the Journal of Biomedical Optics," J. Biomed. Opt. 24(10), 100101 (2019).

6. C. Mack, "Implementing structured abstracts in JM3," J. Micro/Nanolith. MEMS MOEMS 17(3), 030101 (2018).

7. M. T. Eismann, "Structured abstracts," Opt. Eng. 57(11), 110101 (2018). 